\title{
A Large Area Survey for Brown Dwarfs in the Pleiades
}

\author{
Simon Hodgkin \\ Institute of Astronomy, Madingley Road, Cambridge CB3 OHA, UK
}

\begin{abstract}
We present the preliminary findings of a deep imaging survey covering 17 square degrees in the Pleiades. We have identified in excess of 300 candidate cluster members in the $i, i-z$ diagram. The survey is complete to $\sim i=22$, with candidates detected as faint as $i=23$.
\end{abstract}

The Wide Field Camera fills the prime focus of the Isaac Newton Telescope with four $4 \mathrm{k}$ by $2 \mathrm{k}$ EEV42 CCDs. Each chip covers 11 by 22 arcmins with a platescale of 0.33 arcsecs per pixel. One pointing covers 0.27 square degrees (see http://www.ast.cam.ac.uk/ wfcsur).

We have imaged nearly 17 square degrees in the Pleiades with the $i$ and $z$ filters. All data were taken in clear conditions with seeing better than 1.67 arcseconds (median seeing of survey is 1.1 arcseconds). The photometry is calibrated to $5 \%$ or better. The astrometry is accurate to better than 0.1 arcseconds.

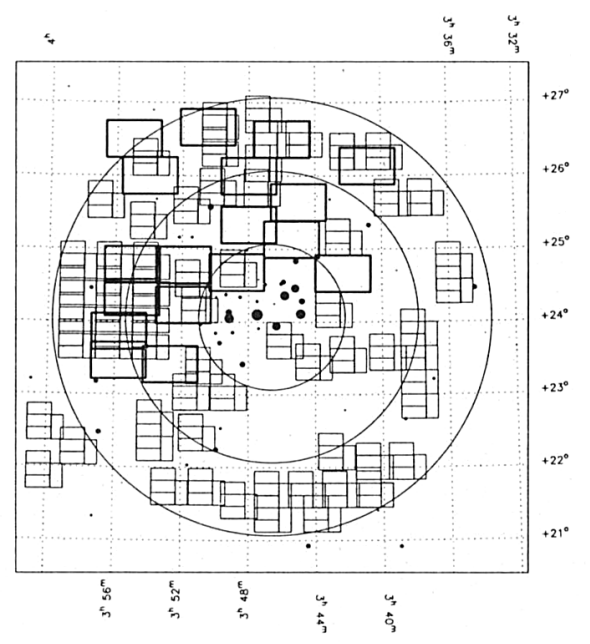

Figure 1. The footprints of the INT Wide Field Camera survey of the Pleiades shown with the brightest members of the cluster. Circles are drawn with radii of 1,2 and 3 degrees. The $18 \mathrm{CFH}$ pointings are also shown (E. Moreaux private communication).

More than 300 candidates are identified from the colour-magnitude diagram. Five nights have been awarded to take JHK photometry of the candidates with UKIRT in November 2002. 


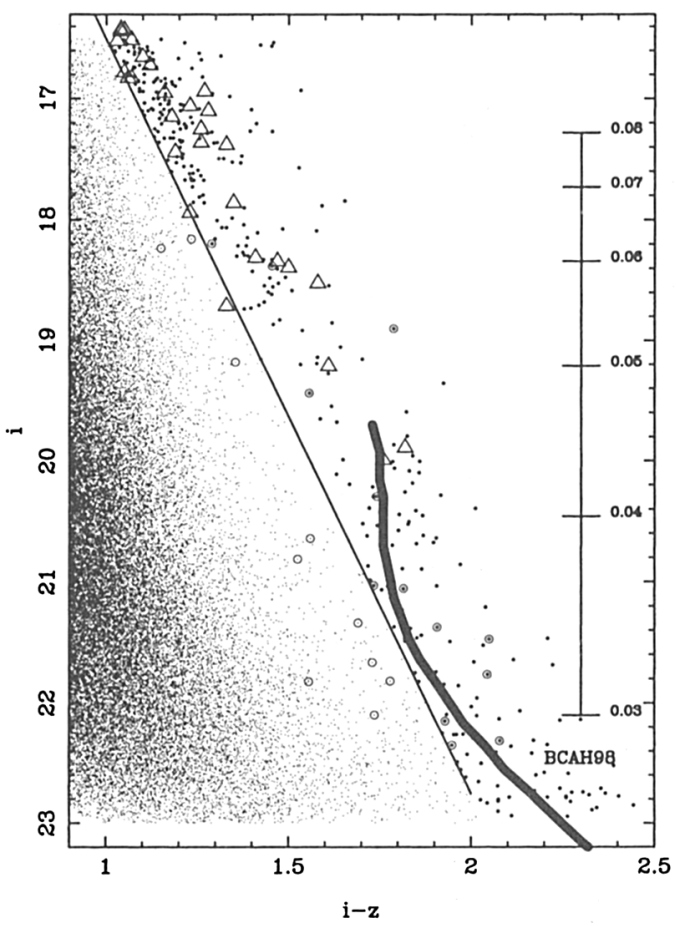

Figure 2. Dots are objects detected in the survey, and the larger dots are the new candidate cluster members. The triangles are previously known cluster members reidentified in the survey. The circles are brown dwarf candidates from the CFH survey (E. Moreaux, private communication). The thick curve shows the expected sequence for Pleiades cluster members (after Dobbie et al. 2002a). The diagonal line shows a preliminary cut for candidate selection. The mass scale comes from Baraffe et al. (1998).

With the clean sample we will (a) Measure the mass function below $30 \mathrm{M}_{\mathrm{Jup}}$. (b) Determine the precise location of the sequence in colour-magnitude and colour-colour space to allow detailed comparison with theory (see e.g. Dobbie et al. 2002b, Jameson et al. this volume). (c) Investigate the binary fraction of the cluster. (d) Examine the spatial distribution of brown dwarfs in the Pleiades.

\section{References}

Baraffe I., Chabrier G., Allard F., Hauschildt P.H., 1998, A\&A, 337, 403

Dobbie P.D., Kenyon F., Jameson R.F., Hodgkin S.T., 2002a, MNRAS, 331, 445

Dobbie P.D., Pinfield D.J., Jameson R.F., Hodgkin S.T., 2002b, MNRAS, in press. 